\title{
Perspectivas y ALCANCES DE LA VINCULACIÓN COMUNITARIA. El CASO DE LA UnIVERSIDAD INTERCULTURAL DE CHIAPAS, UNIDAD OXCHUC
}

\author{
Prospects and Scope of the Community Involvement. The Case of the Intercultural University of Chiapas, \\ Oxchuc Campus
}

\author{
Sergio Iván Navarro-Martínez
}

\begin{abstract}
Resumen: En este artículo se reflexiona sobre las perspectivas y alcances de la vinculación comunitaria en la Unidad Académica Multidisciplinaria de Oxchuc de la Universidad Intercultural de Chiapas (UNICH-Oxchuc). La investigación se realizó entre los años 2013 y 2015 bajo la propuesta metodológica del enfoque etnográfico. Entre los hallazgos destaca que la vinculación comunitaria encuentra limitantes al no establecerse mecanismos de colaboración e interacción sistemática con los actores comunitarios debido a la visión limitada en cómo se aborda y a restricciones burocráticas. Se realizan aportes para pensar la vinculación como un recurso que puede coadyuvar a los procesos comunitarios desde una perspectiva que contemple la experiencia formativa y reflexiva para alcanzar transformaciones.
\end{abstract}

Palabras clave: enseñanza superior, investigación empírica, acción comunitaria.

Abstract: The objective of this article is to discuss the prospects and scope of community outreach undertaken by the Multidisciplinary Academic Unit in Oxchuc, a campus of the Intercultural University of Chiapas (UNICH-Oxchuc). Research was conducted between 2013 and 2015 using an ethnographic methodology. Highlights among the findings include the fact that community outreach is limited, given that ways of systematically interacting with local actors are not in place. This is due to a limited notion about how outreach is done, along with bureaucratic restrictions. The article contributes to revisiting the concept of outreach as a resource that can strengthen community actions based on training and reflection that lead to enacting change.

Keywords: higher education, empirical research, community action.

Sergio Iván Navarro Martínez. Doctor en Ciencias Sociales y Humanísticas por el Centro de Estudios Superiores de México y Centroamérica de la Universidad de Ciencias y Artes de Chiapas, México. Docente en la licenciatura en Planeación del Desarrollo Sustentable en el Centro de Estudios para el Desarrollo Rural, sede Chiapas, México. Temas de especialización: educación intercultural, interculturalidad, vinculación y desarrollo comunitario. Correo electrónico: s-navarro@hotmail.com.
Enviado a dictamen: 28 de noviembre de 2016 .

Aprobación: 9 de mayo de 2017.

Revisiones: 2. 
$\mathrm{E}$ concepto de vinculación ha cobrado relevancia durante los últimos años al cuestionarse el papel que podrían desempeñar las universidades y los centros de investigación frente a los principales problemas sociales, ambientales y productivos de las regiones donde se ubican. Su marcado "academicismo" ha derivado en una tendencia clara de separación entre los procesos de formación y la atención a los problemas - violencia, deterioro ambiental, migración, pobreza, etcétera - que viven de manera cotidiana amplios sectores de la sociedad. En un intento por reorientar en México la política educativa dirigida a los pueblos indígenas, que históricamente han enfrentado situaciones de inequidad en el sistema educativo nacional (Casillas y Santini, 2009: 19), se crearon, a partir del año 2004, las universidades interculturales. En estas instituciones educativas, en las que se pusieron en práctica modelos de educación con enfoque intercultural, una de las principales innovaciones consistió en que los programas de estudio asumen la vinculación comunitaria como una actividad sustantiva. Desde las universidades interculturales:

[...] se plantea la vinculación comunitaria como un conjunto de actividades que implica la planeación, organización, operación y evaluación de acciones en que la docencia y la investigación se relacionan internamente en el ámbito universitario y externamente con las comunidades para la atención de problemáticas y necesidades específicas. Estas actividades [buscan] contribuir al desarrollo de proyectos socioeducativos, culturales y productivos (Casillas y Santini, 2009: 157).

$\mathrm{Al}$ igual que en las demás universidades de este tipo, en la Universidad Intercultural de Chiapas (UNICH) la vinculación es una actividad sustantiva del quehacer educativo para la formación de los jóvenes que ingresan a sus programas. Ávila et al. señalan que la vinculación: "[...] tiene una parte metodológica que permite dotar a los estudiantes de un conjunto de técnicas y herramientas de trabajo participativo que [...] contribuyen indudablemente a generar procesos que van haciendo posible una colaboración estrecha entre la Universidad y la gente de las comunidades" (2016: 769).

En la presente investigación se analiza la experiencia de la licenciatura en Desarrollo Sustentable de la UNICH, Unidad Oxchuc, en la cual se forman jóvenes con la finalidad de contribuir al desarrollo de su comunidad o región, por lo que cabe preguntarse: ¿qué capacidades para la vinculación comunitaria se están generando en los estudiantes?, ¿de qué manera se establecen los vínculos con la comunidad y cómo estos vínculos contribuyen a mejorar la situación de vida de los pobladores?

En el artículo se desarrollan cinco apartados: en el primero se exponen las perspectivas teóricas de la vinculación, en el segundo se presenta el proceso metodológico que guió la investigación, en el tercero se refieren de manera general los antecedentes y la situación actual de la UNICH, en el cuarto se aborda la llegada de esta universidad al municipio de Oxchuc y se describen las características de la licenciatura en Desarrollo Rural y en el quinto se presentan los resultados y discusión de la investigación; se incluye, para finalizar, un apartado de conclusiones.

\section{Perspectivas de la vinculación en las instituciones de educación superior}

Gould plantea que el concepto de vinculación en las Instituciones de Educación Superior (IES) emergió en Estados Unidos en la segunda mitad del siglo XIX para designar cómo los beneficios del conocimiento científico se revertían sobre la sociedad (1997). Sin embargo, fue a principios del siglo XX cuando se impulsó la colaboración del sector educativo con el empresarial, y de la educación y la formación en general con el desarrollo social, económico, científico y tecnológico. "Las contribuciones de la educación superior se dieron en dos áreas generales: primero, la formación de profesionistas y la investigación aplicada; y segundo, las asesorías y el apoyo técnico a la agricultura, el comercio y la industria" (Gould, 1997: 51).

En el caso de México, diversos autores (Acuña, 1993; Casas y Luna, 1994; Romero, 2008) plantean que la 
vinculación entre las IES y el sector productivo comenzó a finales de la década de los ochenta y principios de los noventa, no tanto por el interés de hacer innovación y transferencia tecnológica hacia las industrias, sino más bien de "diversificar las fuentes de financiamiento para la formación de recursos humanos, la investigación científica y tecnológica y el desarrollo de infraestructura institucional" (Casas y Luna, 1994: 1). En consecuencia, las universidades mexicanas respondieron a la necesidad de formar profesionales orientados a la consolidación de un Estado moderno y de estructuras productivas asociadas a un sistema de mercado (Moctezuma y Mungaray, 1996).

Por otra parte, sobresalen experiencias de universidades que han procurado una vinculación orientada hacia la población indígena y campesina, con la finalidad de generar procesos que coadyuven a la sensibilización sobre la realidad en el medio rural y, en algunos casos, a su posible transformación. Ávila et al. (2016) destacan las experiencias desarrolladas por el Departamento de Sociología Rural y la Dirección General de Centros Regionales de la Universidad Autónoma Chapingo, las Brigadas Universitarias de Servicios Comunitarios para la Autogestión de la Universidad Nacional Autónoma de México, el Sistema Modular de la Universidad Autónoma Metropolitana y las estancias de estudiantes realizadas a través de diversas licenciaturas de la Universidad Iberoamericana y el Instituto Tecnológico de Estudios Superiores de Occidente. ${ }^{1}$

Estas experiencias han permitido ampliar el campo de acción de las IES hacia otros sectores que comúnmente no han sido considerados para realizar vinculación. No obstante, "en el caso de las universidades interculturales todavía carecemos de investigaciones monográficas que documenten este importante pilar del quehacer extraáulico en las Universidades Interculturales" (Mateos y Dietz, 2013: 366). ${ }^{2}$

Es importante reconocer que, debido a la influencia mercantilista en la educación, en particular en las últimas décadas se ha puesto énfasis en la interacción de las IES y los centros de investigación con los sectores institucionales, empresariales e industriales
(Gould, 1997; Vallaeys, 1999; Campos y Sánchez, 2005; Malagón, 2006; Alvarado, 2009; Cortez, 2009; Cabrero et al., 2011; Moreno y Maggi, 2011; Saldívar, 2012), lo que responde a un propósito de inserción laboral, más que de formación crítica de los sujetos y de transformación de las condiciones sociales de inequidad e injusticia que prevalecen en las distintas regiones del país.

Para el caso de las universidades interculturales, Dietz propone pactar formas de vinculación directas con el contexto campesino indígena, donde la gestión y producción deben ser asumidas por la universidad desde una perspectiva más académica, con un sentido asociativo y dialógico, es decir "lo que en otros ámbitos [tecnológico o empresarial] se denomina semilleros o incubadoras de iniciativas. Para las universidades interculturales hay que hacerlo en el sentido asociativo, o sea, incubadoras de proyectos, que surjan en el diálogo entre la universidad y la comunidad (Dietz, 2014: 18). ${ }^{3}$ Una perspectiva alternativa a estas visiones consistiría en crear las condiciones para que los estudiantes generen "proyectos de vida colaborativos" que posibiliten su integración en las dinámicas de vida comunitaria, enriqueciendo las experiencias de las familias y de los grupos sociales a partir de los conocimientos adquiridos en la formación y de la recuperación de las experiencias exitosas de la región.

En consecuencia, la generación de capacidades para la vinculación comunitaria, particularmente en las universidades interculturales, debe construirse a partir de posicionamientos reflexivos y críticos en estrecha relación dialógica con las familias, las comunidades, las organizaciones sociales y los actores locales para influir en la consolidación de procesos y proyectos de largo alcance.

\section{Proceso metodológico de la investigación}

En la investigación se utilizaron diferentes métodos y técnicas en momentos distintos que permitieron gradualmente comprender el problema de investigación y profundizar sobre él; por ejemplo, el método etnográfico (Rockwell, 2009) en una primera etapa fue útil para observar de manera participativa, dentro 
y fuera de la institución, los procesos relacionados con la vinculación como práctica docente, a la vez que permitió identificar a informantes clave; se partió del hecho de que "todo trabajo de campo es un ejercicio de observación y de entrevista" (Velasco y Díaz de Rada, 1997: 33).

Se trabajó con una muestra caso-tipo (Hernández, Fernández y Baptista, 2003), con estudiantes de la licenciatura en Desarrollo Sustentable de los semestres sexto, séptimo y octavo, por considerar que en estos niveles los estudiantes han adquirido las competencias teóricas y metodológicas básicas para la vinculación comunitaria y para implementar, si fuera el caso, propuestas de desarrollo comunitario. Se realizaron cuatro grupos de discusión entre los que se aplicaron 32 encuestas cuyos resultados fueron discutidos y reflexionados colectivamente.

Para la encuesta se utilizó la escala de Likert - muy positivo, positivo, regular, negativo y muy negativo-, y fue construida tomando en cuenta los elementos propuestos en los programas de estudio de la licenciatura en Desarrollo Sustentable. En ella se profundizó sobre la forma en cómo se representa y asume la vinculación comunitaria y en experiencia del entrevistado bajo un modelo intercultural. Los resultados se analizaron con la ayuda del programa Statistical Package for the Social Sciences (SPSS) versión 22.

Los grupos de discusión fueron útiles para identificar a los estudiantes que posteriormente se entrevistaron, de manera que se hicieron entrevistas a profundidad a dos estudiantes y semiestructuradas a diecinueve. En cuanto al personal que labora en la UNICH, se realizaron once entrevistas: dos a directivos y nueve a docentes. Paralelamente se acompañó en distintas ocasiones a los estudiantes que realizaban actividades de vinculación en sus comunidades.

Para el análisis de las entrevistas se utilizó el método de comparación constante sugerido por Glaser y Strauss (1967) y por Strauss y Corbin (2002), mediante el cual el investigador simultáneamente codifica y analiza los datos a través de una comparación continua de los incidentes a los que remiten: "lo cual permite desarrollar y refinar conceptos, identificar sus propiedades, explorar sus interrelaciones e integrarlos de una manera coherente" (Wiesenfeld, 2001: 161). Los docentes entrevistados solicitaron guardar el anonimato, lo cual se respeta en este escrito para proteger su identidad, así como la de la totalidad de los entrevistados. El trabajo de campo se realizó, en diferentes etapas, entre agosto de 2013 y junio de 2015.

\section{Antecedentes y situación actual de la UNICH}

La UNICH es la segunda universidad intercultural que se estableció en el país. Se creó por decreto del Congreso de Chiapas en el año 2004 y comenzó a operar en agosto de 2005 en San Cristóbal de Las Casas. En el año 2006 se inició la construcción de las instalaciones ${ }^{4}$ de la Ciudad Universitaria Intercultural en un predio de "10.5 hectáreas, de las cuales 5.5 corresponden a terreno boscoso protegido como área de reserva" (UNICH, 2009: 35). Se encuentra ubicada al sur-poniente de la ciudad, aledaña a la zona de conservación ecológica de los humedales de Montaña María Eugenia.

Según el informe de la administración 2005-2008, la apertura de la UNICHy de sus licenciaturas se realizó tras un proceso de discusión entre intelectuales, académicos y representantes de comunidades y pueblos indígenas, quienes analizaron la pertinencia del funcionamiento de la universidad. "Para tal efecto, la Secretaría de Educación contrató a un despacho especializado para llevar a cabo un estudio de factibilidad que guió la selección de los primeros programas académicos" (UNICH, 2009: 1).

Como resultado de ese trabajo, ${ }^{5}$ se discutieron con la Coordinación General de Educación Intercultural y Bilingüe de la Secretaria de Educación Pública (CGEIPSEP) lasrecomendaciones delestudio para posteriormente abrir cuatro licenciaturas: Lengua y Cultura, Turismo Alternativo, Comunicación Intercultural y Desarrollo Sustentable ${ }^{6}$ que fueron avaladas por la Comisión Estatal de Planeación de la Educación Superior bajo el argumento de que: "no existía otra institución de Educación Superior, pública o privada, en Chiapas, que imparta dichas licenciaturas" (UNICH, 2009: 8).

En el Plan Institucional de Desarrollo 2008-2016 de la UNICH se menciona que uno de los problemas de 
la educación superior en Chiapas es la alta demanda de carreras convencionales, lo que causa saturación en el mercado de trabajo de los egresados; por ello, se enfatiza la necesidad de crear nuevas licenciaturas con pertinencia social y económica.

La iniciativa de la CGEIB-SEP de ubicar las universidades interculturales en localidades rurales o con población mayoritariamente indígena no necesariamente se cumple en la sede central de la UNICH si consideramos que se encuentra en una ciudad que cuenta con más de doscientos mil habitantes. El argumento a favor de su establecimiento en San Cristóbal de Las Casas se basó en el hecho de que la ciudad es un centro regional importante para desarrollar diferentes actividades económicas, sociales, culturales, educativas y turísticas; además de ello, en décadas pasadas las expulsiones por conflictos religiosos contribuyeron a la creación de asentamientos irregulares principalmente de comunidades indígenas en las orillas de la ciudad, ${ }^{7}$ lo que hoy en día genera también demanda de servicios educativos.

Quienes ingresan a la UNICH son jóvenes procedentes tanto de los municipios de la región Altos, ${ }^{8}$ como de la misma ciudad. De acuerdo con los registros, existe una presencia considerable de estudiantes de otras regiones del estado - Meseta Comiteca Tojolabal, Selva Lacandona, Maya, Tulijá Tseltal Chol, Valles Zoque, Metropolitana y Sierra Mariscal-, quienes representan a las principales lenguas indígenas que se hablan en Chiapas (UNICH, 2008). ${ }^{9}$

La universidad amplió su proyecto educativo hacia otras regiones al crear, por un lado, mayores oportunidades para que los jóvenes puedan estudiar en su región y, por otro, para responder a las políticas y estrategias impulsadas por la CGEIB-SEP, es decir: a) ampliar la cobertura de educación superior con pertinencia cultural para la población indígena; b) responder a las demandas indígenas reconociendo los aportes de los pueblos en los aspectos culturales y lingüísticos, y c) que la formación ofrecida se oriente a la solución de problemas y al desarrollo integral de su región (Schmelkes, 2008).

Se crearon otras cuatro sedes o Unidades Académicas Multidisciplinarias (UAM) en distintas regiones de
Chiapas, tres de las cuales iniciaron labores en 2009 con la misma oferta educativa que la ofrecida en San Cristóbal de Las Casas; por ejemplo, en Oxchuc se imparten las licenciaturas en Desarrollo Sustentable, y Lengua y Cultura; en Las Margaritas, Turismo Alternativo, y Lengua y Cultura; en Yajalón, Lengua y Cultura, y Desarrollo Sustentable; y en Valle de Tulijá - Salto de Agua - , que inició labores en agosto de 2012, se ofrecen las licenciaturas en Desarrollo Sustentable y Turismo Alternativo. ${ }^{10}$ En el Plan Institucional de Desarrollo 2008-2016 de la UNICH se reconoce el rezago educativo de la región Sierra; no obstante, ninguna UAM se ha establecido en dicha región.

\section{La licenciatura en Desarrollo Sustentable en la UNICH-Oxchuc}

Antes de que se creara la UNICH en el municipio de Oxchuc sus pobladores habían hecho gestiones ante diferentes instancias de gobierno, sin éxito alguno, para beneficiarse de una IES; en particular deseaban contar con una sede de la Universidad Pedagógica Nacional o una Escuela Normal Superior con la intención de que sus hijos se formaran como maestros de educación primaria. También en diferentes momentos funcionó, por un corto periodo, una IES privada, pero ante la falta de condiciones para establecerse en la zona dejó de operar - los altos costos de las colegiaturas, la falta de reconocimiento oficial de la SEP y la negativa de los pobladores a donarles un terreno para la construcción de sus instalaciones provocaron su cierre-.

Cuando se instaló la UNICH en Oxchuc los habitantes tenían cierto recelo por las experiencias pasadas; sin embargo, su aceptación fue mayor al comprobar que se trataba de una IES con programas de estudios reconocidos por la SEP. En un inicio las autoridades municipales querían que se instalara en terrenos cercanos a la carretera, ${ }^{11}$ pero finalmente los edificios se construyeron en la localidad Piedra Escrita, en un predio donado por las autoridades del municipio.

La UNICH-Oxchuc inició sus labores en agosto de 2009 en las instalaciones del Colegio de Estudios Científicos y Tecnológicos del Estado de Chiapas 
con dos grupos en el turno vespertino, ofreciendo dos licenciaturas - Lengua y Cultura, y Desarrollo Sustentable - dirigidas a los jóvenes de la región que desearan continuar sus estudios. En la primera generación, la difusión se hizo principalmente entre los estudiantes de las preparatorias, pero también entre quienes por alguna situación habían dejado de estudiar. De acuerdo con registros oficiales, la sede de Oxchuc comenzó a funcionar con 68 estudiantes, de los cuales el 95\% eran bilingües - tseltal y español-y el 5\% hablantes pasivos del tseltal, es decir, lo comprendían pero no lo hablaban (UNICH, 2009).

La primera generación (2009-2013) se caracterizó por contar con estudiantes cuya edad no se correspondía necesariamente con el promedio para ingresar a nivel superior, es decir, se matricularon estudiantes que habían concluido su educación media superior años atrás y que por diversas circunstancias tuvieron que interrumpir su proceso educativo. Las generaciones posteriores contaron con estudiantes más jóvenes, recién egresados del bachillerato, y, de acuerdo con testimonios de los docentes, en ninguna de las generaciones se encontraban jóvenes con experiencia laboral, ni en instituciones gubernamentales ni en organizaciones de la sociedad civil; sino que más bien se trataba de jóvenes que habían contado con el apoyo de sus familias para realizar de manera ininterrumpida sus estudios. Lo anterior ofrece elementos para entender parte de las dificultades que los estudiantes han enfrentado para vincularse con los actores comunitarios de la región.

Los programas educativos cuentan con tres niveles: básico, profesional y de especialización; y con cuatro ejes de formación: disciplinar, vinculación con la comunidad, lenguas y sociocultural — todos los mapas curriculares de las licenciaturas de la UNICH tienen los tres últimos ejes-. En el caso de la licenciatura en Desarrollo Sustentable, hay tres líneas de especialización: productiva, ambiental y "buen vivir", ${ }^{2}$ con sus respectivas asignaturas optativas.

Hasta el ciclo escolar agosto-diciembre 2014, la licenciatura en Desarrollo Sustentable contaba con 209 estudiantes inscritos, de los cuales el 93.30\% se reconocían como hablantes de la lengua tseltal y muchos de ellos eran originarios del municipio de Oxchuc, aunque también se contaba con la presencia de estudiantes de otras localidades aledañas como Huixtán, Chanal, Ocosingo, San Juan Cancuc e incluso Cintalapa de Figueroa, municipio de la región Centro de Chiapas.

Desde que contó con instalaciones propias, se estableció como horario de clases de 7:00h a 15:00h de lunes a viernes, y opera bajo un sistema escolarizado. En el tiempo que se realizó la investigación solamente había dos docentes de tiempo completo asignados a la Unidad de Oxchuc, uno para la licenciatura en Desarrollo Sustentable y otro para Lengua y Cultura; los demás fueron contratados por hora, semana o mes, y la mayoría de ellos viajaba desde San Cristóbal a Oxchuc para cumplir con su horario de trabajo. ${ }^{13}$

\section{El acercamiento entre universidad y comunidad}

Como figura en el perfil de egreso, en la licenciatura en Desarrollo Sustentable se busca que los estudiantes dirijan "procesos de investigación participativa para la resolución de problemáticas comunitarias, contribuyendo al bienestar social desde la participación social y política", objetivo que se refuerza desde el eje de vinculación comunitaria a lo largo de la formación profesional.

En el Departamento de Vinculación de la UNICH, situado en la Unidad Central de San Cristóbal, se registró que en 2015 se establecieron acciones de vinculación con cerca de ochocientas comunidades distribuidas en 47 municipios de Chiapas. La información se difundió en las otras unidades académicas para que los docentes encargados del eje de vinculación tuvieran conocimiento de la situación y se evitara, en la medida de lo posible, repetir actividades en las mismas comunidades.

En la revisión documental se identificó que en el proceso educativo se considera la implicación de los estudiantes en la vida comunitaria a través de métodos y técnicas de investigación cualitativas y cuantitativas para propiciar gradualmente procesos de planeación participativa. 
Inicialmente se involucran con la observación y el registro de las diferentes actividades que se despliegan en la comunidad, ${ }^{14} \mathrm{y}$ en los semestres siguientes identifican y jerarquizan problemáticas a partir de pláticas con los actores comunitarios. Esa primera etapa de acercamiento muchas veces se construye con una mirada de afuera hacia adentro, porque es ajena a la realidad cotidiana de la comunidad o familia del estudiante debido a que, paradójicamente, no es una exigencia institucional que éste realice los primeros acercamientos en su propia comunidad, de tal forma que los problemas analizados corresponden a otras realidades, posiblemente parecidas a la suya. ${ }^{15}$

La aprobación para iniciar el trabajo comunitario está bajo la responsabilidad del docente encargado del eje de vinculación, quien considera los siguientes criterios: a) que los estudiantes hablen la lengua materna de la comunidad para generar confianza y facilitar el trabajo; b) los recursos económicos para hacer sus prácticas, pensando que tendrán que realizarlas de manera constante, y c) los hallazgos serán parte del proyecto integrador que presentan al finalizar cada semestre. ${ }^{16}$

Generalmente estos aspectos son considerados en el primer semestre de la licenciatura; sin embargo, en el transcurso de su formación algunos estudiantes cambian de comunidad y dejan a la deriva el proceso de acercamiento construido en los meses anteriores. Esa situación ha ocasionado el descontento y la reticencia de los pobladores para recibir a otros jóvenes que llegan al mismo lugar con similares inquietudes. ${ }^{17}$

En el seguimiento de la vinculación comunitaria que hacen los estudiantes se observó que generalmente se reúnen con grupos ya formados, frecuentemente con los que están integrados a partir de la demanda de alguna institución gubernamental; por ejemplo, con las mujeres del programa Prospera - antes llamado Oportunidades - o con alumnos de alguna escuela primaria. Los estudiantes señalan que en ocasiones trabajan con grupos pequeños de familias ante la falta de interés del resto de la población.

La propuesta educativa de la UNICH pretende contribuir a la solución de los problemas comunitarios, ${ }^{18}$ no obstante, por los hallazgos encontrados se hacen evidentes las dificultades para incidir en los procesos sociales con los que se relacionan los estudiantes durante la vinculación comunitaria. Eso ocasiona que muchos de los actores locales - campesinos, autoridades y líderes, padres y madres de familia, productores, mujeres, jóvenes o docentes, entre otrosno se interesen en participar de manera activa en la formación de los estudiantes.

A pesar de que la UNICH estableció convenios de colaboración con diversas IES e instituciones gubernamentales, ${ }^{19}$ hacen falta mecanismos que permitan fomentar una mayor participación e interacción cotidiana, creativa y dinámica entre la universidad y las comunidades. En este sentido, uno de los docentes entrevistados señaló que: "[...] no se han establecido redes con la comunidad porque no tenemos impacto todavía, no hay ese acercamiento de la universidad con la comunidad y de la comunidad con la universidad, solamente es a través de los alumnos, todavía no hemos aterrizado la relación con las autoridades". ${ }^{20}$

Algunos docentes sugieren que a la hora de trabajar en vinculación con las comunidades no deben promoverse actitudes paternalistas, es decir, no deben ofrecerse insumos a cambio de información, como ocurre bajo las políticas implementadas desde la idea del Estado benefactor, en las que las comunidades reciben "ayuda de los de afuera" - láminas, tinacos, pisos firmes, proyectos productivos, etcétera-, lo que ocasiona que la participación esté condicionada a recibir algo a cambio. Sin embargo, las políticas y las prácticas institucionales contradicen el discurso; por ejemplo, un docente narró lo siguiente: Se supone que con el asunto de la vinculación
comunitaria debe crearse el empoderamiento, pero
la UNICH hizo un convenio con CDI [...] la mayoría
de nosotros no supimos en qué momento se hizo
una consulta con las comunidades para entregarles
instrumentos [musicales], nos dijeron nada más:
"compañeros, tal díanosvemosen Oxchucparaentregar
los instrumentos a las comunidades" [...] después se 
enteraron otras comunidades y nos dieron la solicitud para que les entreguemos instrumentos también. ${ }^{21}$

Esta situación también se reproduce al interior de las aulas; por ejemplo, en la presentación de los proyectos integradores un equipo de estudiantes señaló que, por su propia voluntad, [le] "nació de ir a darle algo [al entrevistado] a cambio de información". Lo anterior puede indicar que, aunque los estudiantes demuestren interés por implementar mecanismos participativos, se ven envueltos en un sistema de dar y recibir a cambio de establecer algún tipo de colaboración u obtener información. Alatorre señala que no siempre se logra una vinculación adecuada debido a la falta de "planeación estratégica [...] o por limitaciones en la capacidad y experiencia de los docentes-tutores", lo que ocasiona que el papel de los estudiantes dentro de las comunidades sea principalmente para extraer información, propiciando "de parte de los miembros de estas comunidades, una respuesta de transacción: iqué vas a darme a cambio?" (Alatorre, 2010: 339).

En una perspectiva innovadora de vinculación social en contextos de diversidad cultural, la preocupación de los estudiantes no debería centrarse en cumplir con las expectativas de las comunidades - proponer obras de infraestructura, proyectos productivos o soluciones inmediatas-, sino en generar procesos reflexivos y de concientización sobre las condiciones históricas injustas de relación con los pueblos indígenas y sobre el papel que éstos podrían jugar en la reivindicación de los modos de ser, estar y convivir en el mundo. Uno de los retos para resignificar la vinculación sería involucrar de manera creativa y complementaria a la universidad con la comunidad, las organizaciones y los actores sociales en iniciativas locales - es decir, sin dependencia de recursos externos - para contribuir a innovar en las actividades que se realizan de forma cotidiana en los contextos comunitarios.

Zemelman plantea que la educación debe aspirar a generar en los “sujetos" la necesidad de hacer algo (Rivas, 2005); en palabras de Restrepo y Rojas, se trata de hacer visible y viable: "la multiplicidad de conocimientos, formas de ser y de aspiraciones sobre el mundo"
(2010: 20-22). Es una apuesta por el reconocimiento del estudiante como actor fundamental de su propio proceso educativo y por la construcción social de sujetos que se colocan frente al mundo, reivindicando su capacidad de transformarlo desde su identidad, historia y contexto socioambiental y productivo.

Los entrevistados reconocen cierta restricción en las formas de organización interna de la comunidad, en las cuales difícilmente un joven universitario puede tener incidencia a la hora de la toma de decisiones. En el siguiente testimonio se encuentra un ejemplo sobre la dificultad de involucrarse en la comunidad:

Cuando fui a comunidad, en primera, reaccionaron que para qué, si no les íbamos a dar nada; pero ya después se dieron cuenta de que éramos alumnos y que necesitábamos formarnos, y dijeron: "Bueno, si les vamos a dar un cambio de mentalidad, creo que es bueno para nosotros", y les ofrecimos el sistema milpa. Hicimos lo de las hortalizas en quinto semestre, pero ahorita ya no hemos hecho nada porque estamos de salida, ya van a venir los de quinto a dar lata con ellos. Nosotros ya no vamos a hacer nada porque ya vamos de salida. ${ }^{22}$

Se identificó que la falta de seguimiento a mediano y largo plazo de los estudiantes a las comunidades con las que se vinculan es un problema central que se debe a dos factores principales: a) la falta de acompañamiento y de sistematización de las actividades que realizan cada semestre en las comunidades - tanto de estudiantes, como de docentes y actores comunitarios - y b) la falta de modelos que les inspiren para promover acciones comunitarias innovadoras; es decir, su arquetipo inmediato, al cual aspiran, es la figura del docente de educación básica y la de los promotores de desarrollo de las instituciones gubernamentales.

Como ya se ha mencionado, a algunos estudiantes les resulta complicado mantenerse en las comunidades seleccionadas debido, en parte, a que generar procesos de vinculación depende en gran medida de los lazos de confianza que se creen en el lugar. En algunos casos logran establecer procesos de reflexión y análisis sobre aspectos 
productivos y sociales con los pobladores de la región; sin embargo, se enfrentan a la falta de confianza en sus propuestas, en gran parte porque son estigmatizados como jóvenes con poca experiencia — esto a pesar de que son de la región y hablan tseltal-.

A partir de estas dificultades, los docentes de la UNICH-Oxchuc realizaron modificaciones de manera interna en el eje de vinculación comunitaria para hacer la intervención más funcional; por ejemplo, en el segundo semestre de 2014 se estableció la semana de la vinculación - una al inicio y otra hacia el final del semestre-, con lo que se buscó subsanar la poca participación de la universidad en las comunidades.

Como se ha señalado, la falta de continuidad y de accesibilidad en algunas comunidades ha derivado en la búsqueda de alianzas con otros actores interesados por las actividades de la UNICH-Oxchuc; regularmente, son los docentes de las escuelas primarias quienes permiten a los jóvenes universitarios poner en práctica parte de los conocimientos adquiridos en la universidad.

Habría que agregar que la compleja situación política del municipio de Oxchuc ha derivado en la suspensión de labores educativas en repetidas ocasiones; una de ellas se produjo durante el movimiento magisterial de 2013, cuando estudiantes y personas del municipio cerraron las instalaciones de la UNICH-Oxchuc en apoyo al magisterio. Otro acontecimiento fue la movilización de la población para exigir la destitución de la presidenta municipal del Partido Verde Ecologista de México que resultó electa en agosto de 2015. Ambas situaciones impidieron llevar a cabo los trabajos de vinculación comunitaria programados para esos semestres.

Por otra parte, es necesario integrar al currículum espacios que permitan reflexionar sobre los retos y alcances que los estudiantes logran en las comunidades semestre tras semestre; esa actividad para sistematizar la experiencia, como señalan Ávila et al., aparece hasta el octavo semestre "para una mejora de sus prácticas" (2016: 770). La falta de espacios de análisis y reflexión sobre su práctica indudablemente puede contribuir al éxito o fracaso de la vinculación comunitaria.

En lainformación recabada en los grupos de discusión se observó que la mayoría de los estudiantes tienen la intención de continuar vinculados a la comunidad, ya sea con proyectos gestionados por ellos mismos o a través de alguna institución gubernamental. Entre sus expectativas destacan las siguientes:

- Trabajar como promotor comunitario haciendo proyectos que salgan desde su comunidad.

- Promover en comunidades locales las nuevas alternativas y estrategias de producción; experimentar y aplicar lo aprendido.

- Gestionar, desarrollar y evaluar proyectos para las comunidades de manera independiente; no estar arraigado a una institución.

- Trabajar con comunidades rurales en proyectos e instituciones como la Secretaría de Agricultura, Ganadería, Desarrollo Rural, Pesca y Alimentos (SAGARPA) o la Secretaría de Medio Ambiente y Recursos Naturales (SEMARNAT).

- Conseguir un trabajo en el cual puedan desarrollar alternativas sustentables.

- Desarrollar proyectos sustentables en las comunidades como, por ejemplo, la preparación de una parcela adecuada con usos y técnicas agroecológicas. ${ }^{23}$

En estas expectativas se observa una enajenación y desvinculación de su lugar de origen, es decir, no se puede negar que a los estudiantes les interesa colaborar con su comunidad, pero sus inquietudes se dirigen hacia construir una colaboración de afuera hacia adentro, es decir, se orientan más a la promoción del desarrollo en su región, sin necesariamente considerar que forman parte de un pueblo. Lo anterior confirma la hipótesis de que prevalecen formas y políticas integracionistas promovidas en décadas pasadas, es decir, la figura del promotor cultural utilizada anteriormente por el Instituto Nacional Indigenista, ahora Comisión Nacional para el Desarrollo de los Pueblos Indígenas, es vigente en el modelo educativo intercultural (Galán y Navarro, 2016). 
Habrá que insistir en que no es fácil lograr que los estudiantes asuman un rol protagónico al interior de su comunidad o región cuando no han tenido experiencias previas de trabajo en organizaciones sociales o comunitarias que les permitan establecer relaciones más sistemáticas al interior de los procesos sociales en los lugares de donde son originarios o en las comunidades donde llevan a cabo los proyectos integradores.

Así, las expectativas institucionales de la UNICH no encuentran mucha correspondencia con las planteadas desde el modelo educativo intercultural (Casillas y Santini, 2009: 157), es decir, las esperanzas de transformación social promovidas por los estudiantes tendrán que esperar un poco mientras no se asuma una política institucional de vinculación como un proceso creativo, sistemático, dinámico y reflexivo con los actores regionales, las organizaciones de la sociedad civil y las autoridades comunitarias y municipales, que haga viable el impulso y el sostenimiento de los proyectos desde una perspectiva intercultural, y que tome realmente en cuenta las marcadas desigualdades históricas y la necesidad de implementar otras formas de relación entre los sujetos.

No obstante, se estimula a los estudiantes para que realicen trabajo en las comunidades:

Hay una mentalidad diferente en los [estudiantes] de Oxchuc, porque están viviendo en un contexto comunitario donde la agricultura, los trabajos comunitarios y los conflictos sociales son fundamentales; y hay todo un concepto de que ser licenciado ya no es vivir en la comunidad, sino vivir fuera del contexto de la oficina y regresar de vez en cuando; si la familia tiene un hijo licenciado tiene mayor estatus dentro de la comunidad. Hemos detectado que están estudiando para trabajar fuera, no están pensando en quedarse en su comunidad. ${ }^{24}$

Aunado a lo anterior, en la encuesta aplicada en los grupos de discusión se preguntó a los alumnos si la formación que reciben en la licenciatura les había permitido proponer soluciones a los problemas de su comunidad. En los resultados, aunque el 33\% efectuó una valoración positiva, sobresale una valoración regular (53.3\%). Un indicador puede ser la falta de trabajo constante en su comunidad.

Las experiencias narradas muestran cómo desde la UNICH-Oxchuc se piensa en la comunidad como en una oportunidad de vinculación y de aprendizaje; no obstante, algunos de los estudiantes no visualizan las comunidades como espacios posibles en los que desarrollar su trabajo, sino que más bien se imaginan trabajando en ellas desde instituciones gubernamentales para promover el desarrollo - de afuera hacia adentro-; en otras palabras, aunque piensan en continuar en estrecha relación - laboral o académicamente - con las comunidades, la mayoría no muestra interés en arraigarse en ellas. Como consecuencia, las capacidades generadas para la vinculación son de carácter inmediatista y funcional, es decir, para la currícula, y están más enfocadas a los resultados que a los procesos.

\section{Conclusiones}

Retomando a algunos autores que han trabajado sobre la perspectiva de la vinculación (Freire, 1984; Gould, 1997; Campos y Sánchez, 2005; Casillas y Santini, 2009; Cortez, 2009; Alatorre, 2010; Saldívar, 2012; Mateos y Dietz, 2013; Dietz, 2014; Ávila et al., 2016; Mateos, Dietz y Mendoza, 2016), considero que la vinculación comunitaria es aquella que busca establecer relaciones de colaboración con los actores y los espacios económicos, socioculturales, ambientales e institucionales del entorno para generar situaciones de aprendizaje y, al mismo tiempo, contribuir a mejorar la situación de vida de la población en general, pero particularmente de la que se encuentra en condiciones de vulnerabilidad.

La propuesta de vinculación de las universidades interculturales ha privilegiado a las comunidades antes que al sector empresarial, de tal forma que se presenta como una innovación del modelo educativo intercultural para incidir en las problemáticas locales. No obstante, por los resultados encontrados, en el caso de la UNICHOxchuc dicha innovación tendría que ir más allá de una práctica agregada a los programas de estudio a través de 
los proyectos integradores, para asumirse como un aporte global al proceso educativo que permita trascender la dependencia institucional y construir formas creativas de relación dinámica y sistemática entre estudiantes, docentes y actores comunitarios. En términos de Freire (1984), se trata de tejer relaciones dialógicas entre los actores para la transformación de la realidad desde una perspectiva crítica.

De igual forma, las reflexiones realizadas permiten plantear que la vinculación comunitaria en la UNICHOxchuc encuentra limitantes porque no se asume la experiencia construida como un aporte esencial al proceso educativo y porque no se establecen mecanismos de colaboración e interacción creativas con los actores comunitarios; estas circunstancias tienen su origen en las restricciones burocráticas, la falta de seguimiento y la visión limitada sobre cómo se aborda la vinculación comunitaria, proceso que debería implicar reciprocidade intercambio de conocimientos para el aprendizaje mutuo.

El desafío consiste en involucrar en el análisis de la realidad, de manera participativa y desinteresada, tanto a los actores comunitarios como a las instituciones y organizaciones sociales y comunitarias, para encontrar intereses compartidos que enriquezcan la germinación de procesos de vinculación comunitaria. Es decir, en la medida en que los estudiantes se involucren de manera gradual en los procesos comunitarios y se fortalezcan sus capacidades teóricas y metodológicas, la vinculación dejará de verse únicamente como una obligación académica.

Es necesario generar interés por conocer qué es la vinculación comunitaria planteada desde la CGEIB-SEP para las universidades interculturales, pero también cabe preguntarse cómo hacer para que las comunidades participen de forma desinteresada en las actividades de vinculación generadas desde la UNICH. Una vía podría consistir en partir de procesos de concientización que posibiliten la incorporación de estudiantes y docentes en las actividades productivas y sociales que se encuentran en marcha en las comunidades, sin que éstos impongan una visión de la realidad, sino más bien contribuyan a potenciar de manera creativa las capacidades e iniciativas sociales.
Se trata de una apuesta por crear diferentes redes entre los actores locales y regionales desde una perspectiva abierta al "diálogo de saberes", ${ }^{25}$ que enriquezca los procesos educativos y comunitarios a través de una "investigación vinculada y vinculante"26 (Alatorre, 2010; Mateos y Dietz, 2013; Mateos, Dietz y Mendoza, 2016; Dietz, 2014). Significa partir de un enfoque educativo intercultural en el que se articule la generación del conocimiento dentro y fuera del aula en colaboración con las comunidades para posibilitar una mayor interrelación entre distintos actores, saberes y disciplinas, "en torno a iniciativas y solidaridades concretas" (Alatorre, 2010: 340).

Por consiguiente, la UNICH-Oxchuc se enfrenta al reto de aportar a la formación para la vida de los estudiantes; es decir, de contribuir a formar sujetos desde una práctica reflexiva en la cual, además, la diversidad sea vista como una oportunidad para enriquecer la cotidianeidad, para reivindicar la identidad y de construir en el presente proyectos de vida colaborativos desde, con y para sus comunidades.

\section{Notas}

${ }^{1}$ Particularmente en Chiapas opera desde 1997 el Programa de Investigación Interdisciplinario Desarrollo Humano de la Universidad Autónoma Metropolitana, que tiene como objetivo investigar en vinculación tanto con los programas de formación de licenciatura y posgrado, como con sectores sociales ligados a la universidad. El programa representa un esfuerzo encaminado a buscar alternativas para mejorar las condiciones de vida de la población en condiciones desfavorables (Cortez, 2009).

${ }^{2}$ Mateos y Dietz mencionan algunos estudios sobre esta temática, por ejemplo el realizado en la UNICH por Gómez y Guzmán, donde se relatan experiencias de la participación de estudiantes en proyectos de desarrollo comunitario; de igual forma, García contribuye con la vivencia establecida entre la UIEP y la comunidad anfitriona a través de un proyecto en conjunto sobre la diversificación productiva. Ebel y Kissmann desarrollan una investigación sobre la sostenibilidad en las comunidades aledañas a la UIMQROO (Gómez y 
Guzmán, s.f.; García, 2009; Ebel y Kissman, 2011, citados en Mateos y Dietz, 2014: 366)

${ }^{3}$ En la década de los setenta Freire ya había realizado una crítica contundente del concepto de "extensión", término utilizado aún en algunas universidades para describir las formasdeinteracciónconsuentorno.Laideade "extensión”, como señala Freire, plantea "llevar" el conocimiento desde las universidades hacia las comunidades y es una acción con "una connotación indiscutiblemente mecanicista" (Freire, 1984: 26), que no pasa por una reflexión crítica de cómo se construye la relación entre los sujetos. Para superar esta carga de imposición, sugería el concepto de "comunicación", en la perspectiva de reconocer el papel dialógico que deberían contener las relaciones entre los actores para la transformación de la realidad desde una perspectiva crítica.

${ }^{4}$ Una de las críticas fuertes en torno a la construcción de las instalaciones de la UNICH parte de que su diseño arquitectónico denota la influencia colonial, aspecto que precisamente la interculturalidad busca erradicar.

${ }^{5}$ A partir de la investigación realizada se constató que, como tal, no hay estudio de factibilidad, por lo que es sugerente pensar que las licenciaturas fueron creadas en función de los intereses de la CGEIB-SEP (Bermúdez, 2015), debido a que se encuentra una similar oferta educativa en otras universidades interculturales, a excepción de la Universidad Veracruzana Intercultural y de la Universidad Indígena Autónoma de México, cuyos programas educativos no necesariamente se ajustan a los criterios del modelo intercultural.

6 Situaciones similares se encuentran en otras universidades interculturales; por ejemplo, Rojas, Navarro y Escobar (2016) demuestran que en la Universidad Intercultural del Estado de Tabasco no huboun estudio de factibilidad y se abrieron únicamente las licenciaturas propuestas por la CGEIB-SEP.

${ }^{7}$ Rus identifica tres olas de migrantes que llegaron a San Cristóbal. La primera sucedió en la década de los setenta: "conducida por jóvenes bilingües que querían cambiar de religiónyestablecersefuera de sus comunidades de origen, y la segunda se conformó principalmente de refugiados económicos atraídos por las nuevas oportunidades de vivienda y empleo abiertas por la primera ola; la tercera consistió cada vez más en refugiados a secas, presionados tanto por condiciones económicas adversas como, después de 1994, por la violencia” (Rus, 2012: 225).

${ }^{8}$ Es conocida oficialmente como Región V Altos Tsotsil y Tseltal. Está conformada por diecisiete municipios, su índice de analfabetismo es del $26.8 \%$, y el $68 \%$ de la población en la región es hablante de alguna lengua indígena (Programa Regional de Desarrollo, s.f.).

${ }^{9}$ Tras la última regionalización de 201l, Chiapas cuenta con quince regiones socioeconómicas.

${ }^{10}$ Las unidades académicas que han ampliado su oferta educativa son las de San Cristóbal de Las Casas, con la carrera de Médico Cirujano, junto con Oxchuc y Las Margaritas con la licenciatura en Derecho Intercultural.

${ }^{11}$ El pueblo de Oxchuc está dividido por la carretera que comunica el municipio con Ocosingo y Palenque; en ambos lados de la carretera se encuentran diferentes servicios de transporte y comercios.

${ }^{12}$ El "buen vivir" se plantea como una alternativa a la visión economicista del desarrollo para buscar una relación más armónica y respetuosa entre los seres humanos y la naturaleza; significa equidad e igualdad tanto en las relaciones sociales, como en la distribución de recursos (Ávila, 2012; Cevallos, 2012).

${ }^{13}$ De acuerdo con los datos de la coordinación académica de la UNICH-Oxchuc, en el segundo semestre de 2014 laboraban un total de veintiún docentes, de los cuales once contaban con estudios de licenciatura, ocho de maestría -incluyendo los dos docentes de tiempo completo- y uno de doctorado.

${ }^{14}$ Para iniciar la vinculación comunitaria, los estudiantes se presentan ante las autoridades comunitarias, ejidales o educativas para dar a conocer su plan de trabajo previamente revisado por los docentes.

${ }^{15}$ En las entrevistas con los estudiantes y docentes, éstos se refieren a que desde el primer semestre se integran los equipos de vinculación comunitaria, y en cada uno de ellos participa por lo menos un estudiante de esa comunidad, quien la mayoría de las veces asume un rol protagónico porque conoce el contexto y a los actores locales. Por otra parte, esto puede ser una desventaja para el resto de los integrantes del equipo cuando ese estudiante decide cambiar de comunidad. 
${ }^{16}$ Para Peña, Marín y Camacho (2015: 9), el proyecto integrador es un documento que articula los conocimientos transversales y disciplinares del aula con los conocimientos locales, y, en el caso de sexto y séptimo semestre, representa un anteproyecto de titulación. La información encontrada se presenta en el aula al finalizar cada semestre.

17 Por ejemplo, en la materia "Taller de planeación comunitaria participativa", de quinto semestre, se observó que un grupo de jóvenes identificó algunos obstáculos en su proyecto de educación ambiental, el cual consistía en hacer un huerto demostrativo familiar. Ante la falta de seguimiento al proyecto, y por no contar con participación de la comunidad, decidieron cambiarse de localidad y abandonaron el proceso iniciado.

18 Tania Cruz Salazar, en su investigación "El joven indígena en Chiapas: el re-conocimiento de un sujeto histórico", señala que los egresados tienen una responsabilidad mayor con su comunidad ya que "tendrá[n] que diseñar, gestionar y promover proyectos productivos o de gestión cultural, crear trabajos en su comunidad, detectar problemáticas y organizar a la gente para que en grupo busque la forma de autoemplearse y generar los propios insumos para su vida cotidiana y la de los suyos iVaya tarea les ha tocado a estos jóvenes!" (Cruz, 2012: 157).

${ }^{19}$ Entre ellos: la Escuela Superior de Educación de Coimbra Portugal, el Instituto de Capacitación y Vinculación Tecnológica, las dependencias de turismo de Chiapas, la COPARMEX San Cristóbal de Las Casas, la Universidad Autónoma Metropolitana, el Gimnasio de Arte y Cultura A.C.,laUniversidaddePoitiers, laUniversidaddeLimoges, el Centro Latinoamericano de Gestión Ambiental A.C., la Secretaría de Pueblos y Culturas Indígenas, la Universidad Politécnica de Chiapas, la UNAM y la CDI con tres convenios específicos: "Levantamiento y captura de informe sobre 28 planes micro-regionales", "Realización de diagnóstico alimentario-nutricional, en el marco de la Cruzada Nacional Contra el Hambre" y "Elaboración de proyectos identificados en los planes micro-regionales" (UNICH, 2013).

${ }^{20}$ Entrevista a docente de la UNICH-Oxchuc.
${ }^{21}$ Entrevista a docente de la UNICH-Oxchuc.

${ }^{22}$ Entrevista a estudiante de la UNICH-Oxchuc.

${ }^{23}$ Grupo de reflexión con estudiantes de la UNICHOxchuc.

${ }^{24}$ Entrevista a docente de la UNICH-Oxchuc.

${ }^{25}$ El diálogo de saberes no sólo establece un espacio de sinergias y complementariedades entre los saberes existentes sobre el mundo actual y la realidad presente, sino que apunta a la producción —más que a la generatividad óntico-epistémica-científicatecnológica- de nuevas formas de comprensión del mundo que emergen de la dialógica del intercambio de saberes y de la disputa de sentidos en la reapropiación social de la naturaleza y de la cultura (Leff, 2003: 33).

${ }^{26}$ Se entiende como "el reconocimiento de las diferentes epistemologías y de la diversidad de conocimientos, técnicas, sistemas de valores y aspiraciones de los grupos sociales inmiscuidos en la relación universidad/ comunidad; segundo, en la utilización de los recursos, las tecnologías, los saberes y los sistemas tradicionales de organización locales como referentes para la construcción de conocimiento significativo con el que operar en la realidad; tercero, en la valoración, la comprensión y el respeto por los contenidos de las culturas y las lenguas locales, así como la promoción de una pedagogía para la afirmación de la propia cultura pero que asuma como igualmente válidas otras lógicas culturales. Por consiguiente, su pertinencia se sustenta en el conocimiento profundo de la historia y la cultura de la región, y se instrumenta en acciones con un fuerte componente de vinculación comunitaria" (Ávila et al., 2014: 666).

\section{Referencias}

Acuña, Patricia (1993). "Vinculación universidad-sector productivo". En Revista de la Educación Superior, 22(87): 1-15.

Alatorre, Gerardo (2010). "La instrumentación de un modelo educativo basado en el diálogo y la investigación vinculada con actores comunitarios. Logros, tropiezos y retos de la Universidad Veracruzana Intercultural." En: Saúl Velasco Cruz y Aleksandra Jablonska Zaborowska (coords.) Construcción de políticas educativas interculturales 
en México: debates, tendencias, problemas, desafíos. México: Universidad Pedagógica Nacional.

Alvarado, Aida (2009). "Vinculación universidad -empresa y su contribución al desarrollo regional". En RaXimhai, 5(3): 407-414.

Ávila, Agustín (2012). "II. El buen vivir: una alternativa a la crisis civilizatoria actual”. En Sartorello, Stefano y León Ávila (coords.), El buen vivir: miradas desde adentro de Chiapas. México: UNICH, Gobierno del Estado, UNESCO, IESAIC.

Ávila, León, Verónica Moreno, Rodolfo Escobar, Óscar Trujillo y Héxtor Bernardino (2014). "El reconocimiento de los saberes locales en la cuenca hidrográfica transfronteriza Grijalva como punto de partida para el desarrollo de capacidades de manejo ambiental". En Mario González Espinosa y Marie Claude Brunel Manse (coords.), Montañas, pueblos y agua: dimensiones y realidades de la Cuenca Grijalva. México: El Colegio de la Frontera Sur y Juan Pablos Editor.

Ávila, León, Alberto Posada, Gabriela Arias y Agustín Ávila (2016). "Vinculación comunitaria y diálogo de saberes en la educación superior intercultural en México". En Revista Mexicana de Investigación Educativa, 2(70): 759-783.

BermúdezUrbina, Flor Marina (2015). "Interculturalidad, etnicidad e intermediación cultural en la formación de profesionales indígenas. Concepciones y prácticas en la Universidad Intercultural de Chiapas". En Patricia Medina (coord.), Sujetosy conocimientos situados, políticas del lugar en educación. Trayectos y experiencias pedagógicas de investigación en la construcción de interculturalidades activas. Oaxaca: 1450 Ediciones, UNACH, UABJO, UNICH, UPN.

Cabrero, Enrique, Sergio Cárdenas, David Arellano y Edgar Ramírez (2011). "La vinculación entre la universidad y la industria en México". En Perfiles Educativos, XXXIII, número especial.

Campos, Guillermo y Germán Sánchez (2005). "La vinculación universitaria: ese oscuro objeto del deseo". En Revista Electrónica de Investigación Educativa, 7(2).

Casas, Rosalba y Matilde Luna (1994). "Condicionantes políticos de la nueva relación entre universidad e industria”. En M. Campos y L. Corona (coords.),
Universidad y vinculación: nuevos retos y viejos problemas. México: IIMAS-UNAM

Casillas, Lourdes y Laura Santini (2009). Universidad Intercultural: modelo educativo. México: SEP-CGEIB.

Cevallos, Francisco (2012). Educación y buen vivir: reflexiones sobre su construcción. Quito: Contrato Social por la Educación

Cortez, Carlos (2009). "El vínculo universidad-sociedad en la experiencia del Programa de Investigación Interdisciplinario Desarrollo Humano de la Universidad Autónoma Metropolitana, México". En Daniel Mato (coord.), Educación superior, colaboración intercultural y desarrollo sostenible/buen vivir. Experiencias en América Latina. Caracas: Instituto Internacional de la UNESCO para la Educación Superior en América Latina y el Caribe.

Cruz Salazar, Tania (2012). "El joven indígena en Chiapas: el re-conocimiento de un sujeto histórico". En LiminaR. Estudios Sociales y Humanísticos, X (2), juliodiciembre: 145-162.

Dietz, Gunther (2014). "Diversidad e interculturalidad en la universidad: logros y desafíos". En Instituto Nacional Electoral (ed.). Democracia intercultural, Voz de expertos. México: INE.

Freire, Paulo (1984). ¿Extensión o comunicación? La concientización en el medio rural. México: Siglo XXI.

Galán, Felipe y Sergio Navarro (2016). "Indigenismo y educación intercultural: una discusión necesaria. La experiencia en la Universidad Intercultural del Estado de Tabasco". En Desacatos. Revista de Ciencias Sociales, 52: 144-159.

Glaser, Barney y Anselm Strauss (1967). El desarrollo de la teoría fundada. Chicago: Aldine.

Gould, Giacomo (1997). Vinculación universidad-Sector productivo. Una reflexión sobre la planeación y operación de programas devinculación. México: ANUIES, Universidad Autónoma de Baja California.

Hernández, Roberto, Carlos Fernández y Pilar Baptista (2003). Metodología de la investigación. La Habana: Editorial Félix Varela.

Leff, Enrique (2003). "Racionalidad ambiental y diálogo de saberes: sentidos y senderos de un futuro sustentable", Desenvolvimento e Meio Ambiente, 7: 13-40. 
Malagón, Luis (2006). "La vinculación universidadsociedad desde una perspectiva social". En Revista Educación y Educadores, 9(2): 79-93.

Mateos, Laura, Gunther Dietz y Rosa Mendoza (2016). "iSaberes-haceres interculturales? Experiencias profesionales y comunitarias de egresados de la educación superior intercultural veracruzana". En Revista Mexicana de Investigación Educativa, 21(70), 809-835.

Mateos, Laura y Gunther Dietz (2013). "Universidades Interculturales en México". En María Bertely, Gunther Dietz y G. Díaz (coords.), Multiculturalismo y educación 2002-2011. México: Consejo Mexicano de Investigación Educativa, A.C., ANUIES.

Moctezuma, Patricia y Alejandro Mungaray (1996). La reorganización universitaria orientada por la vinculación. Un estudio de caso. México: ANUIES.

Moreno, Maricruz y Rolando Maggi (2011). "Estrategias de vinculación de las universidades mexicanas con las empresas". Ponencia presentada en el XI Congreso Nacional de Investigación Educativa. Política y Gestión, Ciudad de México, 7 a ll de noviembre de 2011.

Peña-Cuanda, María, Carmen Marín y Angélica Camacho (2015). "Género, lenguas originarias y vinculación comunitaria: Una apuesta de transversalidad en las curriculas a nivel superior para apuntalar la educación intercultural". Ponencia presentada en el Congreso Latinoamericano de Americanistas, El Salvador Conflicto Paz e Identidades, 12 al 17 de julio de 2015.

Programa Regional de Desarrollo. Región V Altos Tsotsil Tseltal (s.f.). Disponible en: http://www.haciendachiapas. gob.mx/planeacion/Informacion/DesarrolloRegional/proregionales/ALTOS.pdf (consultado el 5 de febrero de 2017).

Restrepo, Eduardo y Axel Rojas (2010). Inflexión decolonial: fuentes, conceptos y cuestionamientos. Colombia: Instituto de Estudios Sociales y Culturales Pensar, Maestría en Estudios Culturales-Universidad Javeriana.

Rivas, Jorge (2005). "Pedagogía de la dignidad de estar siendo. Entrevista a Hugo Zemelman y Estela Quintar". En Revista Interamericana de Educación de Adultos, 27: 113-140.
Rockwell, Elsie (2009). La experiencia etnográfica: historia y cultura en los procesos educativos. Buenos Aires: Paidós.

Rojas, Victoria, Sergio Navarro y Amelia Escobar (2016). "Historia de la UIET, su génesis y situación actual". En Revista Saberes de los Pueblos, 1(1): 15-29.

Romero, José (2008). Experiencias de vinculación universidadsector productivo y social de la BUAP. México: ANUIES.

Rus, Jan (2012). El ocaso de las fincas y la transformación de la sociedad indígena de los Altos de Chiapas 1974-2009. México: CESMECA-UNICACH.

Saldívar, Antonio (2012). "Educación superior, desarrollo y vinculación sociocultural. Análisis de experiencias educativas universitarias en contextos rurales e indígenas". Tesis de doctorado, Universidad Carlos III de Madrid, España.

Schmelkes, Sylvia (2008). "Las universidades interculturales en México: ¿Una contribución a la equidad en educación superior?" Ponencia presentada en la First Conference on Ethnicity, Race, and Indigenous Peoples in Latin America and the Caribbean, Universidad de California, 22 a 24 de mayo de 2008, San Diego, California.

Strauss, Anselm y Juliet Corbin (2002). Bases de la investigación cualitativa: técnicas y procedimientos para desarrollar la teoría fundamentada. Medellín: Universidad de Antioquia.

UNICH (2008). Plan Institucional de Desarrollo 2008-2016. Chiapas, México: UNICH.

UNICH (2009). Informe Institucional 2009. Chiapas, México: UNICH.

UNICH (2013). Informe Institucional 2013. Chiapas, México: UNICH.

Vallaeys, François (1999). "El desafío de enseñar ética en la universidad”. En Papeles de Ética, Economía y Dirección, 4.

Velasco, Honorio y Ángel Díaz de Rada (1997). La lógica de la investigación etnográfica. Madrid: Trotta.

Wiesenfeld, E. (2001). La autoconstrucción, unestudiopsicosocial del significado de la vivienda. Venezuela: Universidad Central de Venezuela-Facultad de Humanidades y Educación y Comisión de Estudios de Postgrado 